\title{
Horizontal Wind Systems in the Ionospheric $E$ Region Deduced from the Dynamo Theory of the Geomagnetic $S_{q}$ Variation Part III
}

\author{
By Hiroshi MAEDA \\ Department of Earth Science, Yoshida College, Kyoto University \\ (Read July 5, 1957 ; Received July 10, 1957)
}

\begin{abstract}
In Parts I and II ionospheric wind systems have been deduced from the geomagnetic $S_{q}$ variations for the mean solstice. In order to compare our results with observation, however, it is still necessary to obtain the wind systems for the solstitial seasons. In this paper wind systems in summer and winter hemispheres are deduced from the $S_{q}$ data. It is shown that the diurnal wind component has greater magnitude in summer than in winter; whereas the semi-diurnal component has smaller magnitude in summer than in winter over most of the hemisphere, and that in summer hemisphere the diurnal wind component is greater than the semi-diurnal one; whereas in winter hemisphere the former is more or less smaller than the latter. A comparison between our results and observed ionospheric winds is made.
\end{abstract}

\section{Introduction}

In Part I (by this author [1]) and Part II (by S. Kato [2]) of this report we have obtained the horizontal wind systems in the ionospheric $E$ region from the dynamo theory of the geomagnetic $S_{q}$ variation, and it has been found that the main component of winds required to produce the $S_{q}$ variations is not semi-diurnal, but diurnal. M. Hirono and T. Kitamura [3] have also treated this problem on some different assumptions, and obtained the results similar to ours. Since all these works [1,2,3] are based on geomagnetic data for the mean solstice, the results seem to represent a mean state of summer and winter. In order to compare the calculated results with observation, it is, therefore, still necessary to obtain the wind systems for each of both solstitial seasons. In the present paper (Part III) the wind systems in Northern summer and winter (i.e. Southern winter and summer, respectively) are deduced from the geomagnetic data by a similar method to in Part I, on the assumption, for simplicity, that the motion of the air in regions concerned is horizontal and irrotational. Further extension of Part III, taking into account the effect of the Coriolis force, will be made in Part IV by Kato [4].

The data used are the horizontal intensity of the geomagnetic $S_{q}$ variations at 62 stations over the world in summer and winter of the Second Polar Year, 1932-33. The 
harmonic coefficients of the North $(\Delta X)$ and East $(\Delta Y)$ components of $S_{q}$ are plotted in Fig. 1. The full (for Northern summer) and dotted (for Northern winter) lines in this figure show a smooth distribution of the coefficients derived from the potential of the $S_{q}$ field for the mean solstice [5] and solstitial difference [6] (see Table 1), on the assumption that the mean solstitial field of $S_{q}$ is symmetrical, and that the solstitialdifference field is anti-symmetrical with respect to the equatorial plane. Since the spherical harmonic coefficients shown in Table 1 are obtained from the $S_{q}$ data in the interzonal region (between $60^{\circ} \mathrm{N}$ and $60^{\circ} \mathrm{S}$ ), the high-latitude curves in Fig. 1 are an extraporation of $S_{q}$ in middle and low latitudes.

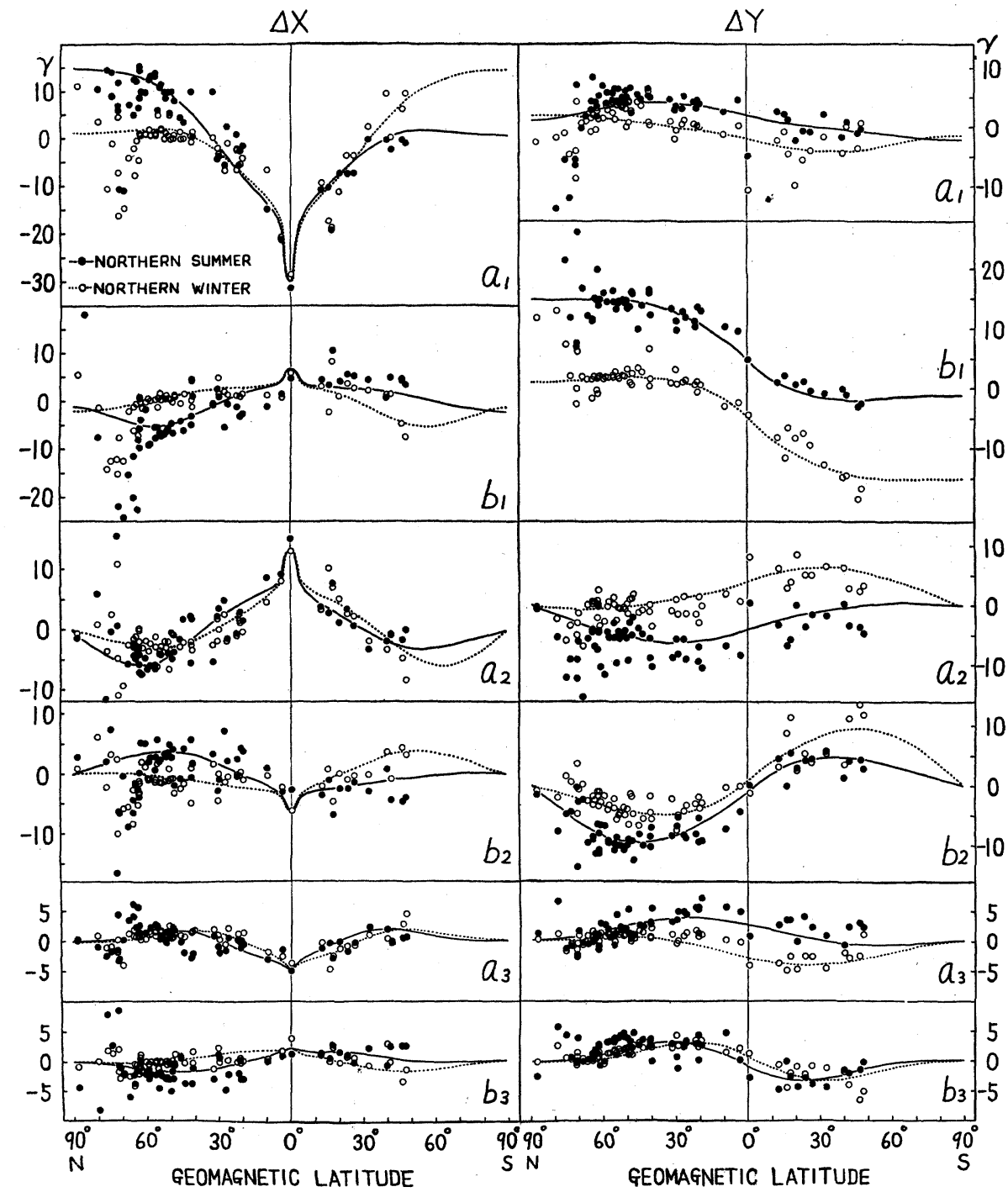

Fig. 1 Latitude-distributions of the harmonic coefficients for the geomagnetic North $(\Delta X)$ and East $(\Delta Y)$ components of the geomagnetic $S_{q}$ variations in summer and winter. The full (for Northern summer) and dotted (for Northern winter) lines show a smooth distribution obtained from the spherical harmonic coefficients listed in Table 1. 


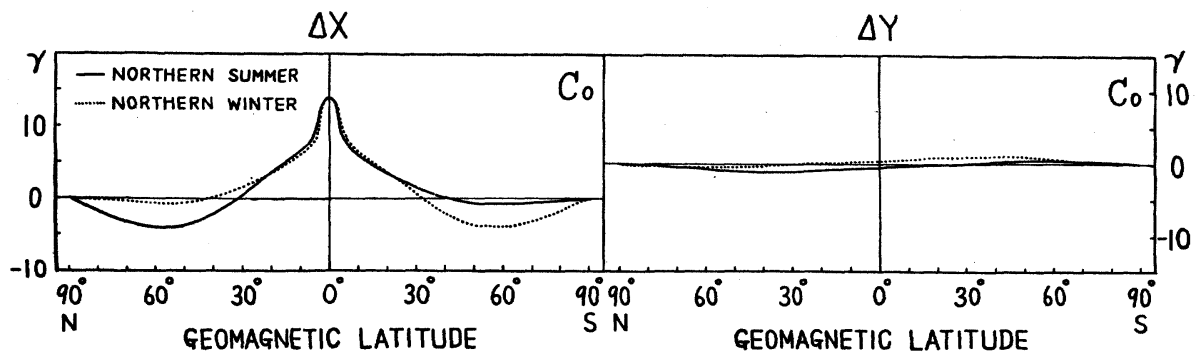

Fig. 2 Latitude-distributions of the constant term $C_{0}$.

Table 1. Spherical harmonic coefficients for the mean solstitial and solstitial-difference fields of $S_{q}$ in the interzonal region (between $60^{\circ} \mathrm{N}$ and $60^{\circ} \mathrm{S}$ ) analyzed in the form

$$
V=r_{0} \sum_{n} \sum_{m}\left(a_{n}^{m} \cos m t+b_{n}^{m} \sin m t\right) P_{n}^{m}(\cos \theta),
$$

using the Second Polar Year data. (Unit $=r)$

$$
\frac{1}{2}(S+W) \quad \frac{1}{2}(S-W)
$$

\begin{tabular}{|c|c|r|r||r|r|r|}
\hline$m$ & $n$ & $a_{n}^{m}$ & $b_{n}^{m}$ & $n$ & $a_{n}^{m}$ & $b_{n}^{m}$ \\
\hline \multirow{3}{*}{1} & 2 & 8.40 & -2.35 & 1 & 5.41 & -1.90 \\
& 4 & -1.75 & 0.40 & 3 & 0.82 & 0.43 \\
& 6 & 0.21 & 0.12 & 5 & -0.14 & 0.34 \\
\hline \multirow{3}{*}{2} & 3 & -4.15 & 1.69 & 2 & -0.88 & 2.38 \\
& 5 & 0.14 & -0.15 & 4 & -0.67 & 0.03 \\
& 7 & -0.02 & 0.22 & 6 & -0.10 & -0.08 \\
\hline \multirow{2}{*}{3} & 4 & 1.42 & -0.86 & 3 & -0.19 & -1.16 \\
& 6 & 0.30 & -0.02 & 5 & 0.27 & 0.04 \\
\hline
\end{tabular}

\section{Method}

In this paper, the dynamo equation eliminating the velocity potential is used. Omitting unnecessarily detailed descriptions (see [1] or [3]) we have

$$
\begin{aligned}
\frac{\partial^{2} S}{\partial \theta^{2}}+\frac{1}{\sin \theta \cos \theta} \frac{\partial S}{\partial \theta}+\frac{1}{\sin ^{2} \theta} \frac{\partial^{2} S}{\partial \lambda^{2}} & =-a\left\{\frac{\partial E_{x}}{\partial \theta}+\frac{E_{x}}{\sin \theta \cos \theta}+\frac{1}{\sin \theta} \frac{\partial E_{y}}{\partial \lambda}\right\} \\
& \equiv F(\theta, \lambda),
\end{aligned}
$$

where $S$ is the electrostatic potential ; $\theta, \lambda$ the colatitude and east longitude, respectively; $a$ the radius of a current sheet; $E_{x}, E_{y}$ the Southward and Eastward components of the total electric field.

The right-hand side of (1) can be calculated, if the total electric field $\boldsymbol{E}$ is known. This field is approximately estimated from the relation

$$
\left.\begin{array}{l}
I_{x}\left(\simeq \frac{\Delta Y}{2 \pi}\right)=\Sigma_{x x} E_{x}+\Sigma_{x y} E_{y}, \\
I_{y}\left(\simeq \frac{\Delta X}{2 \pi}\right)=-\Sigma_{x y} E_{x}+\Sigma_{y y} E_{y},
\end{array}\right\}
$$

using the geomagnetic North $(\Delta X)$ and East $(\Delta Y)$ components of $S_{q}$, corrected by the constant term $C_{0}$ (see Fig. 2), and the height-integrated conductivities given by the form [ 7 ] 
where

$$
\left\{\begin{array}{l}
\Sigma_{x x} \\
\Sigma_{y y} \\
\Sigma_{x y}
\end{array}\right\}=\left\{\begin{array}{l}
\Sigma_{x x 0} \\
\Sigma_{y y 0} \\
\Sigma_{x y 0}
\end{array}\right\}\left(1+2.00 \cos \chi+1.46 \cos ^{2} \chi\right)
$$

$$
\chi=\text { solar zenith angle. }
$$

The total field for the Northern summer (i.e. Southern winter) is shown in Fig. 3.

Thus the differential equation (1) for the electrostatic potential $S$ can be solved. The boundary conditions satisfied by $S$ are $S$ must vanish at the poles, and the static field derived from $S$ is equal to the total field at the equator, because the dynamo field vanishes there.

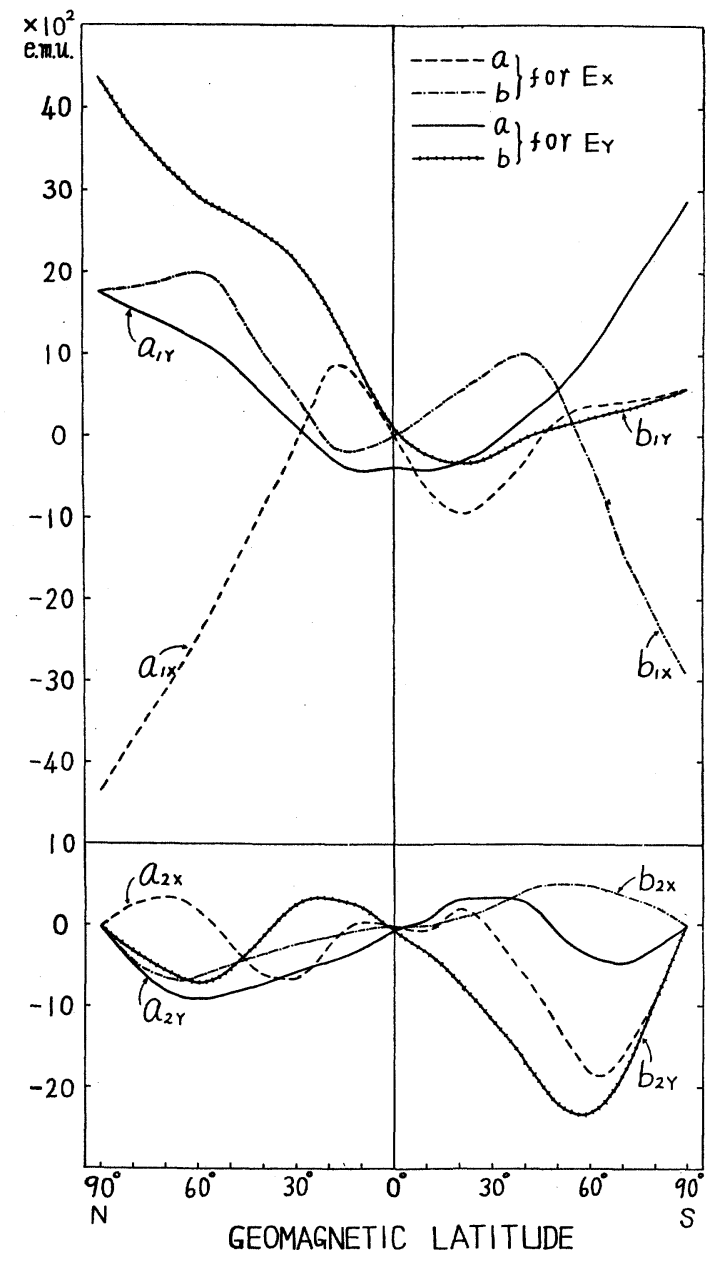

Fig. 3 Latitude-distributions of the harmonic coefficients for the total electric field components $E_{x}, E_{y}$ in Northern summer.

\section{Solution of the Differential Equation}

The right-hand side of ( 1 ) calculated by using the total field obtained above is expressed as follows:

$$
F(\theta, \lambda)=\sum_{m}\left\{f_{m a}(\theta) \cos m \lambda+f_{m b}(\theta) \sin m \lambda\right\} .
$$

If we put 


$$
S(\theta, \lambda)=\sum_{m}\left\{s_{m a}(\theta) \cos m \lambda+s_{m b}(\theta) \sin m \lambda\right\},
$$

then (1) is reduced to

$$
\left.\begin{array}{l}
\frac{\partial^{2} s_{m n}}{\partial \theta^{2}}+\frac{1}{\sin \theta \cos \theta} \frac{\partial s_{m a}}{\partial \theta}-\frac{m^{2}}{\sin ^{2} \theta} s_{m n}=f_{m a}(\theta), \\
\frac{\partial^{2} s_{m h}}{\partial \theta^{2}}+\frac{1}{\sin \theta \cos \theta} \frac{\partial s_{m b}}{\partial \theta}-\frac{m^{2}}{\sin ^{2} \theta} s_{m b}=f_{m b}(\theta) .
\end{array}\right\}
$$

The general solution of (3) consists of a particular solution and complementary functions. A particular solution $s_{m(p)}$ is obtained by means of numerical integration. The homogeneous equation of $(3)$ is reduced to the hypergeometric equation by the same substitutions as in Part I. One of linearly independent solutions of this equation tends to infinity as $\theta \rightarrow 0$, hence only the following solution is adopted:

$$
s_{m(1)}=\sin ^{m} \theta F\left(\frac{m}{2}, \frac{m}{2} ; m+1 ; \sin ^{2} \theta\right),
$$

where $F(a, b ; c ; z)$ stands for the hypergeometric function. The general solution is, therefore, given by

$$
s_{m}=s_{m(p)}+C s_{m(e)},
$$

where $C$ is an integration constant. Since both particular and complementary solutions satisfy the conditions at the poles, the value of integration constant $C$ can be determined by the condition at the equator, consequently the unique solution is obtained. Further detailed descriptions of the method of obtaining the solution will be given in Part IV by Kato [4].

\section{Results}

The static field $\boldsymbol{E}_{s}$ is derived from the potential $S$ as follows:

$$
E_{s x}=-\frac{\partial S}{a \partial \theta}, \quad E_{s y}=-\frac{\partial S}{a \sin \theta \partial \lambda},
$$

and subtraction of this field from the total field gives the dynamo field $\boldsymbol{E}_{\boldsymbol{i}}$. Thus the wind velocity is calculated from the dynamo field by the relation

$$
u=-\frac{E_{i y}}{H_{z}}, \quad v=\frac{E_{i x}}{H_{z}},
$$

where $H_{z}$ is the vertical component of the geomagnetic intensity. The resultant distributions of the wind velocity are illustrated in Figs. 4 and 5 for each of Northern summer and Northern winter. The wind vectors of the diurnal and semi-diurnal components shown in Figs. 4 and 5 are also plotted in Fig. 6 in polar form, for convenience of comparison with observation.

From these figures the following will be seen:

\section{(a) Diurnal wind component}

1) The 24-hourly harmonics represent a clockwise rotation in the Northern hemisphere, and an anticlockwise rotation in the Southern hemisphere. 2) The movement 
is towards the North (South) at $1500 \mathrm{hr}$ in summer in the Northern (Southern) hemisphere, and at $1100 \mathrm{hr}$ in winter in the Northern (Southern) hemisphere. 3) The magnitude of the rotating vectors is about $40 \mathrm{~m} / \mathrm{sec}$ in summer, and about $15 \mathrm{~m} / \mathrm{sec}$ in winter,

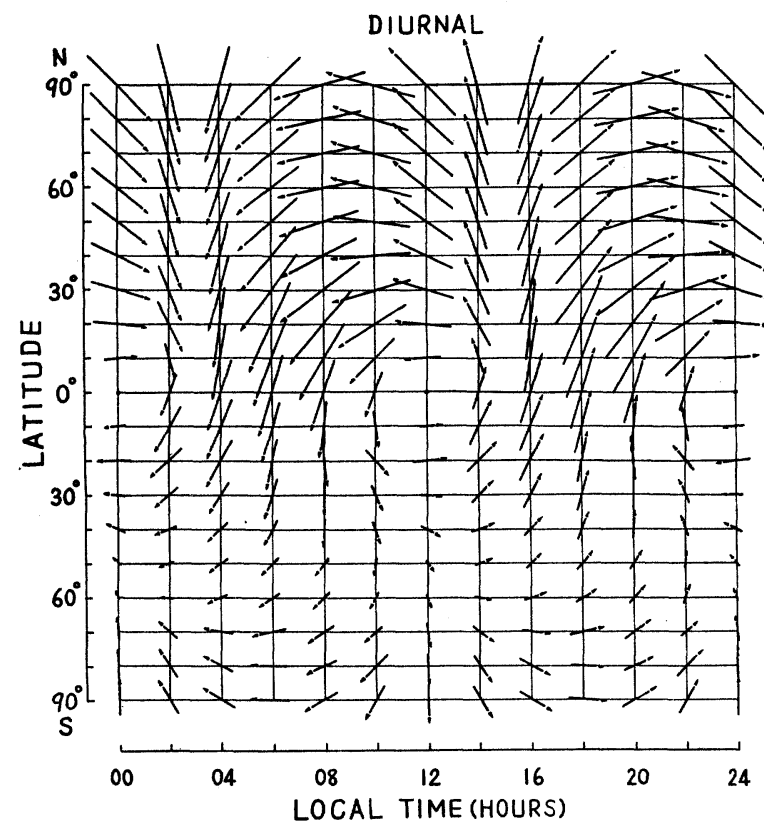

SEMI-DIURNAL
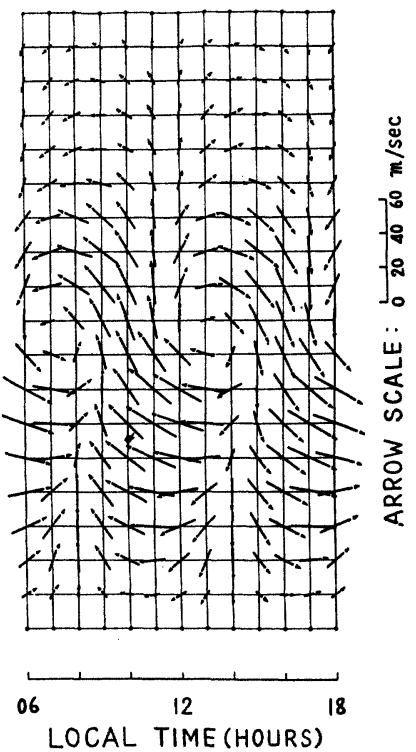

Fig. 4. Distributions of the wind velocity in Northern summer for each of the diurnal (left) and semi-diurnal (right) components.

\section{DIURNAL}

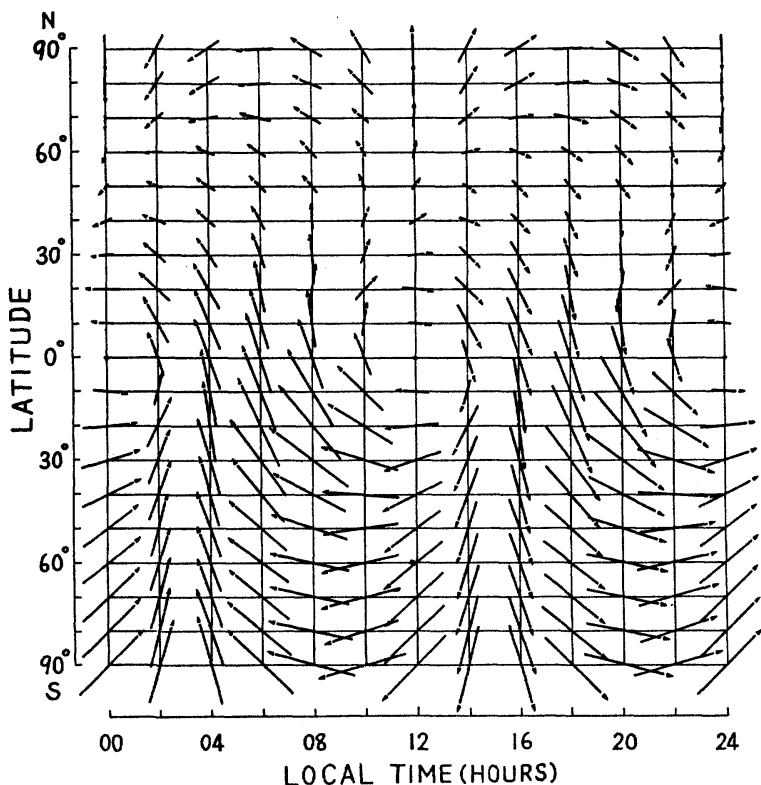

SEMI-DIURNAL

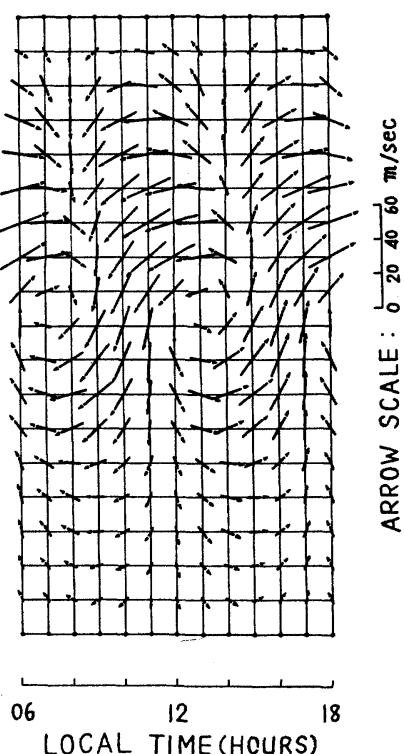

Fig. 5 Distributions of the wind velocity in Northern winter for each of the diurnal (left) and semi-diurnal (right) components. 
for both hemispheres.

\section{(b) Semi-diurnal wind component}

1) The 12-hourly harmonics represent a clockwise rotation in the Northern hemisphere, and an anticlockwise rotation in the Southern hemisphere. 2) The movement is towards the North (South) at 1130 and $2330 \mathrm{hr}$ in summer in the Northern (Southern) hemisphere, and at 0230 and $1430 \mathrm{hr}$ in winter in the Northern (Southern) hemisphere. 3) The magnitude of the rotating vectors is about $10 \mathrm{~m} / \mathrm{sec}$ in summer, and about $20 \mathrm{~m} / \mathrm{sec}$ in winter, for both hemispheres.
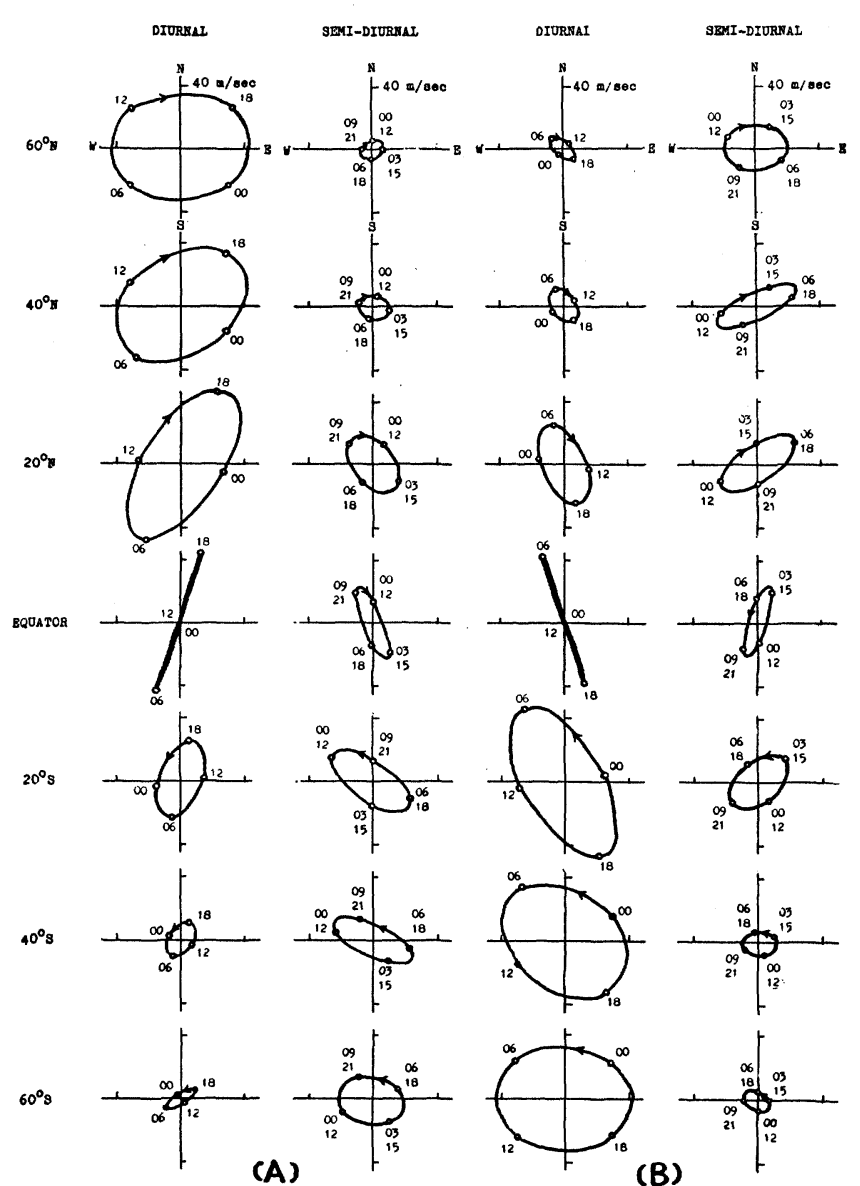

MORTHERE SORARR (SOUTHBa WIHTBR)

AORTHERN WIKTEB (

Fig. 6 Polar plots of the diurnal and semi-diurnal wind components at latitudes of $0^{\circ}, 20^{\circ} \mathrm{N}$ and $\mathrm{S}, 40^{\circ} \mathrm{N}$ and $\mathrm{S}$, and $60^{\circ} \mathrm{N}$ and $\mathrm{S}$, in Northern summer (A) and in Northern winter (B).

\section{Summary and Discussion}

The wind systems required to produce the geomagnetic $S_{q}$ variations in Northern summer and winter are deduced from the dynamo theory. The wind vector associated with both harmonic components rotates clockwise in the Northern hemisphere, and anti- 
clockwise in the Southern hemisphere. This direction of the rotation and the magnitude of the rotating vectors are in general agreement with the observed results [8] (for both harmonics) [9, 10] (for semi-diurnal). However, the phase of the rotation does not agree with the observed one. The observed phase is such that the diurnal wind component blows towards the South near $0600 \mathrm{hr}$ in the Southern hemisphere [8], and that the semi-diurnal wind component blows towards the North (South) near 0600 and $1800 \mathrm{hr}$ in the Northern (Southern) hemisphere $[8,10]$. The difference between the calculated and observed phases may be accounted for by the fact that the winds at altitudes of $80-100 \mathrm{~km}$ which are probably lower than the dynamo region are measured by the radio-echo meteor technique.

The diurnal wind vectors have greater magnitude in summer than in winter, whereas the semi-diurnal wind vectors have smaller magnitude in summer than in winter over most of the hemisphere. And, in summer hemisphere the diurnal component of winds is greater than the semi-diurnal one, whereas in winter hemisphere the former is more or less smaller than the latter. This situac.un seems to be consistent with the results observed by L. Harang and K. Pedersen [11]. For the sake of exact comparison, however, further systematic measurements of ionospheric winds, especially in the dynamo region $(100-130 \mathrm{~km})$, are still necessary.

\section{Acknowledgments}

The author wishes to express his sincere thanks to Prof. M. Hasegawa and Prof. K. Maeda for their valuable discussions and advice, and to Prof. Y. Tamura and Dr. M. Ota for their continued interest and support in the course of this study. He is also very grateful to Mr. A. Kijima who had kindly taken the trouble to check our solution of the differential equations by Integro-Differential Analyser.

\section{References}

[1] H. Maeda, J. Geomag. Geoelectr., 7, 121 (1955).

[2] S. Kato, J. Geomag. Geoelectr., 8, 24 (1956).

[3] M. Hirono and T. Kitamura, J. Geomag. Geoelectr., 8, 9 (1956).

[4] S. Kato, J. Geomag. Geoelectr., 9, 107 (1957).

[5] H. Maeda, J. Geomag. Geoelectr., 5, 39 (1953).

[6] M. Hasegawa and M. Ota, Read at the Meeting of the Japanese Society of Terrestrial Magnetism and Electricity, Oct. 16, 1956.

[7] “H. Maeda, Rep. Ionosph. Res. Japan, 10, 49 (1956).

[8] W. G. Elford and D. S. Robertson, J. Atmosph. Terr. Phys., 4, 271 (1953).

[9] B. H. Briggs and M. Spencer, Phys. Soc. Rep. Prog. Phys., 17, 245 (1954).

[10] J.S. Greenhow and E. L. Neufeld, Phil. Mag., 46, 549 (1955).

[1.1] L. Harang and K. Pedersen, J. Geophys. Res., 62, 183 (1957). 